\title{
AWARENESS AND ATTITUDES OF PEOPLE WITH DIABETES REGARDING THEIR DISEASE IN HYDERABAD AND ADJOINING AREAS
}

\author{
Asif A. Burney, Mansoor A. Khowaja, Javed I. Memon, M. Sadik Memon, A. Akbar Siddiqui \\ and Imran A. Sheikh
}

\begin{abstract}
BACKGROUND/OBJECTIVES: Diabetes Mellitus (DM) is a chronic disease, associated with high degree of morbidity and mortality. Together with the advances in management of DM, and the widespread availability of treatment, patient's education and awareness about the disease is equally important for the optimum management of the disease. Hence, this study was designed to explore the prevailing attitudes and awareness of diabetic patients in and around Hyderabad - Sindh.

DESIGN: A cross sectional survey.

PATIENTS AND METHODS: Interviews were made regarding attitude, awareness and education about the disease. Percentage scores were then compared separately among the gender (2 groups: male and female), age of the patients (3 groups; group 1, 18-30 years, group II, 31-50 years and group III 51-80 years), and time since the diagnosis of DM (3 groups; group A, less than 6 months, group B, 6.1 month to 3 years, and group C 3.1 years and more).

RESULTS: Total 127 patients were interviewed including 88(69.3\%) men and 39(30.7\%) women. Among these, $\mathbf{5 1 . 7 \%}$ men and $\mathbf{5 1 \%}$ women had a positive attitude while $55.4 \%$ of men and $\mathbf{3 8 . 8 \%}$ women had adequate awareness. Also, $68.8 \%$ men and $62.8 \%$ women were adequately educated. A positive attitude was observed in $4.8 \%, 46.8 \%$ and $46.1 \%$ respectively in group I, II and III patients respectively. Corresponding figures for awareness were $49.6 \%, 55.1 \%, 44.4 \%$ and for education $63.2 \%, 69.4 \%$ and $65.1 \%$. Regarding relationship to the chronicity of the disease, a positive attitude was observed in $53.8 \%$ of group A patients, $47.7 \%$ and $41.4 \%$ amongst group B and $C$ patients respectively. Corresponding figures for awareness and education were $41.1 \%$, $49.1 \%$ and $56.2 \%$ and $57.8 \%, 64.2 \%$ and $7.7 \%$ and $57.8 \%$ respectively.

CONCLUSION: Adequate awareness and education about diabetes is lacking. Some aspects of diabetes education need more emphasis. Diabetes education should be tailored according to the individual patient understanding.
\end{abstract}

KEY WORDS: Knowledge. Attitude. Diabetes Mellitus. Developing Countries.

\section{INTRODUCTION}

Diabetes Mellitus (DM) is a chronic disease, associated with high degree of morbidity and mortality. ${ }^{1-3}$ It is one of the major health problems around the world. The prevalence of DM in the province of Sindh, Pakistan is reported up to $13.9 \%{ }^{4}$ Together with the advances in the management of DM, and the widespread availability of treatment, patient's education and awareness about the disease is equally important for the optimum management of disease. ${ }^{5}$ By optimum control of DM, this may help to avoid or delay the short and long-term complications of diabetes. Studies have shown that attitudes, beliefs and awareness about the disease play very important role in the glycemic control and the onset of chronic complications of diabetes mellitus. ${ }^{6-7}$ Most of the chronic complications of diabetes are not curable. Collectively, these complications are responsible for majority of cardiovascular, cerebrovascular, renal, nervous, foot and eye disease burden in the community. Baseline knowledge about the nature of the disease should be available to the patients with diabetes. Diabetic patients should be more precisely knowledgeable about the role of diet and exercise to help control this metabolic syndrome. These patients should be adequately edu- 
cated about the chronic complications well before these complications appear. Some of these chronic complications are present even at the time of diagnosis in some type 2 diabetes patients. Patient education about the disease should therefore start with the onset of treatment as soon as a diagnosis of diabetes is established. A regular and frequent interaction between the patient and the caregiver is required to accomplish these goals. Together this would help in the better management of the patients with diabetes. The purpose of this study was to explore the prevailing attitudes, level of awareness and education of diabetic patients in and around Hyderabad city of Sindh province of Pakistan. Hence, strategies can be developed to promote health education by giving more emphasis to areas where gaps are found in the knowledge of the patients in our setup.

\section{PATIENTS AND METHODS}

This was a cross sectional study, conducted on a sample of convenience. Study was conducted at Isra University Hospital Diabetes Clinic and at the private clinics of Isra University Hospital physicians between July 1, 2004 and December 31, 2004. Isra University and Hospital are located in the outskirts of Hyderabad, the fifth largest town of Pakistan. The catchment area of Isra University Hospital mainly includes the urban population of Hyderabad city and the rural population residing in small towns and villages alongside the national highway and the districts of Tando Allahyar and Mirpurkhas. Patients coming to the private clinics of the physicians of Isra University are mostly city dwellers. Collectively, this comprises a heterogeneous population especially in terms of ethnicity and literacy. An informed consent was obtained from all patients who filled the questionnaire. Consenting patients between the ages 18 and 80 years, and with a firm diagnosis of DM type 1 or 2 (fasting blood sugar level more than $126 \mathrm{mg} \%$ on two separate occasions) were interviewed. The questionnaire contained 34 variables including demographics and information about different aspects of the DM. Most of the questions were replied in 'YES' or 'NO' and the average time taken to fill the questionnaire was 12 minutes. Inquiry was made regarding the attitude, awareness and education about the disease. Questions regarding the attitude (taking the problem seriously or not) towards the disease included: frequency of consultation (weekly, fortnightly or monthly- in this study the correct answer was fortnightly) and blood sugar monitoring (within a week), medicine and dietary compliance, employing other treatment options and self- medication, exercise and smoking. Questions about the awareness (does the patient understand appropriately what (s)he has been told) included: proper understanding of the diet, exercise and various chronic complications of diabetes mellitus. Regarding the education (had the patient ever been told about the problem), questions were asked about diet, exercise and eye and foot care. Total 9 questions were asked about the attitude, 7 questions were related to awareness and 4 questions were asked about the education. Answers to the questions were considered either correct or incorrect. One mark was given in case of being correct and no marks were given for incorrect answers. Correct answers of all patients were added in sections of attitude, awareness and education separately and a percentage score of total number of patients was obtained in every case. The percentage scores were then compared separately according to the gender (2 groups: male and female), age of the patients (3 groups; group I, 18-30 years, group II, 31-50 years and group III, 51-80 years), and time since the diagnosis of DM (3 groups; group A, less than 6 months, group B, 6.1 month to 3 years, and group C, 3.1 years and more). Data were analyzed using SPSS version 11. Descriptive statistics were computed for data presentation. Significance level was taken at $p<0.05$.

\section{RESULTS}

Out of total 127 patients interviewed, 88 (69.3\%) were male and 39 (30.7\%) females. Regarding gender, it was found that $51.7 \%$ of male patients and $51 \%$ of the female patients had a positive attitude towards the disease, based on the sample of questions asked $(p=0.810)$. Overall, $55.4 \%$ of males had adequate awareness of the disease. On the contrary, only $38.8 \%$ of female patients had adequate awareness $(p=0.000)$. However, $68.8 \%$ of males were adequately educated about diabetes at some time in the course of their disease in comparison to $62.8 \%$ of females who were adequately educated $(p=0.190)$. Regarding different age groups, $43.8 \%$ of the group I patients had a positive attitude towards the disease, whereas, in groups II and III, a positive attitude was observed in $46.2 \%$ and $46.1 \%$ respectively $(p=0.859)$. In group I, $49.6 \%$ had adequate awareness, of group II $55.1 \%$ were adequately aware, whereas, the level of ade- 
Asif A. Burney, Mansoor A. Khowaja, Javed I. Memon, et al.

quate awareness was $44.4 \%$ in group III patients $(p=0.013)$. Regarding education, $63.2 \%$ of patients of group I, $69.4 \%$ of patients of group II and $65.1 \%$ of patients of group III were adequately educated about the disease $(p=0.505)$. Awareness, attitude and level of education were also studied according to the duration of disease and findings are shown in Table I. Patients of group A had a positive attitude in $53.8 \%$ of cases. For group B patients, the level was $47.7 \%$, whereas, only $41.4 \%$ showed positive attitude amongst the group $C$ patients $(p=0.003)$. Adequate level of awareness was exhibited by $41.1 \%, 49.1 \%$ and $56.2 \%$ of patients in groups $A, B$ and $C$ respectively $(p=0.001)$. Level of education was found to be appropriate in $57.8 \%, 64.2 \%$, and $73.7 \%$ of patients in groups $A, B$ and $C$ respectively $(p=0.006)$. Eighteen $(14.2 \%)$ cases out of 127 patients checked their blood sugar regularly i.e. within seven days. Fourty - two (33.1\%) patients were using unconventional treatment modalities such as, herbal medicine etc. in addition to insulin or oral hypoglycemic agents, whereas 15 (11.81\%) patients were using unconventional methods of treatment only. Fourty-one (32.28\%) patients had at least a fortnightly consultation either at the diabetic clinic or at a physician's private clinic. Majority of patients i.e. $110(86.61 \%)$ first went to a physician on having their diabetes discovered.Fourty - six (36.22\%) patients smoked. Majority of patients i.e.108(85.03\%) were explained about the diet in detail at some time of their disease, whereas 96 (75.59\%) patients recalled having been explained about the exercise in detail. Meanwhile, 66 (51.96\%) patients were told about the importance of routine eye examination and 70 (55.11\%) were ever explained about the foot care. tion is required to promote better understanding of DM, thus achieving better glycemic control among the diabetic patients. This subsequently leads to a reduction in morbidity and mortality from the disease. ${ }^{8} \mathrm{Ap}-$ propriate education provided by health care professionals can modify the beliefs about the disease and fill the gaps in patient's knowledge. ${ }^{9}$ Hence, it is also important to establish the level of understating about DM in a cross-section of people. This study was designed to assess the level of awareness, attitudes and existing level of education about DM in various sets of diabetic patients in Hyderabad and adjoining areas. Hyderabad is the fifth largest town of Pakistan. Two studies of similar nature have been conducted in Quetta, Balochistan and North West of Pakistan. Both studies included only type 2 diabetes patients and having diabetes for more than one and two years respectively. ${ }^{10-11}$ On the contrary, this study included both type 1 and 2 diabetic patients and also having a recent diagnosis of diabetes mellitus. We found that the attitude of women towards the disease was comparable to that in the male patients. Similarly, the level of education amongst female patients was comparable to male patients. However, there was a significant difference about the awareness of the disease in women as compared to men who demonstrated adequate awareness. This dichotomy suggests that although knowledge was equally dispatched to the female patients yet the level of awareness they achieved was significantly less than that of the male patients. Whether this low level of awareness was due to low literacy rate in the females, less frequent visits of female patients to the physicians, poor communication with the female gender by the physicians and

TABLE I: AGE DISTRIBUTION AND TIME SINCE DIABETES IS KNOWN

\begin{tabular}{|l|c|c|c|c|}
\hline \multirow{2}{*}{ Age in years } & \multicolumn{3}{|c|}{ Time since diabetes is known } & Total number of \\
patients
\end{tabular}

\section{DISCUSSION}

Effective communication and disease specific educa- health-care professionals, or other elucidated factors, need further exploration. However, the overall level of 
awareness in this study was found to be better than the study conducted by Ali, et al in Quetta Balochistan, where only $22.9 \%$ of diabetic patients displayed adequate level of awareness about their disease. ${ }^{10}$ It was interesting to note that the older patients were less aware of their disease as compared to the younger patients. Even more interesting observations were made when the awareness, attitudes, and level of education were studied in comparison to the chronicity of disease. When the level of education increased with the advancing disease the overall attitude towards the disease got worse over time. This dichotomy may be related to the longevity of the disease process, lack of interest on the part of the patient as the disease progress, and forcing too many changes in diet and lifestyle by the physicians, to which the patients may fail to comply. Lack of positive attitude with advancing disease has significant deleterious implications, and need to be studied further. This study does not support the observations of Hasan, et al, where they did not find significant decline in attitude or better understanding of the disease with the passage of time. ${ }^{11}$ We found that patients were adequately educated about diet and exercise, yet only about half of the patients were educated about foot care and importance of routine eye examination. Yet, the level of education about the foot care is better as compared to the results seen in study by Hasan, et al where only a negligible percentage of diabetic patients had the foot care. These areas need special consideration in diabetic patient education since diabetes is the leading cause of foot amputation and blindness. ${ }^{12}$ Thirty-three percent of patients tried traditional and herbal medicines at sometime during the course of their disease. This is in conformity to the observations of Al-Saeedi, et al who also found that one third of patients used traditional medicines. ${ }^{13}$ In contrast, a study conducted in India has showed the use of alternative medicines in diabetics by two third of patients. ${ }^{14}$ Similarly, a study conducted in rural Mexico about the knowledge and beliefs in diabetic patients has revealed the use of home remedies in majority of the subjects. ${ }^{15}$

\section{CONCLUSION}

There is much more to be done in the area of diabetes education in our setup. Some aspects of diabetes education need more emphasis than others. Diabetes education should be tailored according to the individual patient understanding.

\section{REFERENCES}

1. Powers AC. Diabetes mellitus. In Braunwald E, Fauci AS, Kasper DL, Hause SL, Longo DL, Jameson JL, (eds). Harrison's principles of internal medicine. New York: McGraw-Hill, 2001; Pp. 2109-37.

2. Shera S. Diabetes: a major health problem of Pakistan. Diabetes Digest. 1992; 5: 7-15.

3. Zimmet P. Diabetes: definitions and classification. Med Int. 1997; 11:1-3.

4. Shera AS, Rafique G, Khwaja IA, Ara J, Baqai S, King $\mathrm{H}$. Pakistan national diabetes survey: prevalence of glucose intolerance and associated factors in Shikarpur, Sindh province. Diabet Med. 1995; 12:1116-21.

5. Afridi MA, Khan MN. Role of health education in the management of diabetes mellitus. J Coll Physicians Surg Pak. 2003;13:558-61.

6. Hawthorne K. Effect of culturally appropriate health education on glycemic control and knowledge of diabetes in British Pakistani women with type 2 diabetes mellitus. Health Educ Res. 2001; 16: 373-81.

7. Harris R, Linn MW. Health beliefs, compliance and control of diabetes mellitus. South Med J. 1985; 78:162-6.

8. Diabetes Control and Complications Trial (DCCT) Research Group. N Engl J Med.1993; 329:977-86.

9. Smith SM, O'Leary M, Bury G. A qualitative investigation of the views and health beliefs of patients with type 2 diabetes following the introduction of a diabetes share care service. Diabet Med. 2003; 20: 853-7.

10. Ali M, Khalid GH, Pirkani GS. Level of health education in patients with type-2 diabetes mellitus in Quetta. J Pak Med Assoc. 1998; 48:334-6.

11. Hasan ZU, Zia S, Maracy M. Baseline disease knowledge assessment in patients with type 2 diabetes in a rural area of northwest of Pakistan. J Pak Med Assoc. 2004; 54:67-73.

12. Paul Riordan-Eva, Eye. In: Tierney, Mcphee, Papadakis. Current medical diagnosis and treatment. McGraw-Hill. 2004; Pp. 145-74.

13. Al-Saeedi M, Elzubeir AG, Bahnassi AA. Patterns 
Asif A. Burney, Mansoor A. Khowaja, Javed I. Memon, et al.

of belief and use of traditional remedies by diabetic patients in Mecca, Saudi Arabia. East Mediterr Health J. 2003; 9:99-107.

14. Mahrotra R, Bajaj S, Kumar D. Use of complementary medicine by patients with diabetes melli- tus. Natl Med J India. 2004;17:243-5.

15. Valenzuela GA, Mata JE, Mata AS, Gabali C. Knowledge and beliefs regarding type 2 diabetes mellitus in rural Mexico. Ethn Health. 2003; 8: 353-60.

AUTHOR AFFILIATION:
Dr. Asif Ali Burney (Corresponding Author)
Associate Professor, Department of Medicine
Isra University Hospital Hala Road P.O. Box \# 313,
Hyderabad, Sindh, Pakistan.
Email: docasif_65@hotmail.com.
Dr. Mansoor A. Khowaja
Department of Community Medicine
Isra University Hala Road, Hyderabad - Pakistan.
Dr. Javed I. Memon
Department of Medicine
Isra University Hospital, Hyderabad - Pakistan.
Dr. M. Sadik Memon
Department of Medicine
Isra University Hospital, Hyderabad - Pakistan.
Dr. A. Akbar Siddiqui
Department of Medicine
Isra University Hospital, Hyderabad - Pakistan.
Dr. Imran A. Sheikh
Department of Medicine
Isra University Hospital, Hyderabad - Pakistan.
Dra

